\title{
Scenario Mining in the Urban Domain: Exploiting the Topology of a Road Network for Maneuver Annotation and Scenario Extraction
}

This paper was downloaded from TechRxiv (https://www.techrxiv.org).

\section{LICENSE}

CC BY-SA 4.0

SUBMISSION DATE / POSTED DATE

$11-02-2022$ / 16-02-2022

\section{CITATION}

Klitzke, Lars; Schicktanz, Clemens; Gimm, Kay; Koch, Carsten; Köster, Frank (2022): Scenario Mining in the Urban Domain: Exploiting the Topology of a Road Network for Maneuver Annotation and Scenario Extraction. TechRxiv. Preprint. https://doi.org/10.36227/techrxiv.19161755.v1

DOI 


\title{
Scenario Mining in the Urban Domain: Exploiting the Topology of a Road Network for Maneuver Annotation and Scenario Extraction
}

\author{
Lars Klitzke®*, Clemens Schicktanz®*, Kay Gimm $\odot^{*}$, Carsten Koch $^{\dagger}$ and Frank Köster ${ }^{\ddagger}$
}

\begin{abstract}
Before Automated Vehicles (AVs) can enter the market, academic and industrial partners must tackle system homologation to ensure the functional safety of AVs. For that task, a scenario-driven approach has gained acceptance in recent years since it allows facing the overall system's complexity. Real-world drivings are a valuable source of scenarios. However, their extraction and analysis are still the subjects of research. This work addresses this issue and proposes a novel methodology to extract routing scenarios on urban intersections. Therefore, trajectories are annotated with routing maneuvers using a semantically enriched road network and map matching for route backtracking. The accuracy of the approach and consistency of the methodology is evaluated with Brunswick's inner-city ring and by extracting u-turn scenarios on the Application Platform for Intelligent Mobility (AIM) Research Intersection of the German Aerospace Center (DLR).

Index Terms-Scenario Mining, Naturalistic Driving Data, Unsupervised Learning, Map Matching, Route Backtracking, Automated Vehicles
\end{abstract}

\section{INTRODUCTION}

Although Automated Vehicles (AVs) are envisioned to transform future mobility [1], their functional safety must be verified before being released into the public domain. That is a major challenge because AVs need to operate in a complex and constantly changing environment. Therefore, several research projects such as PEGASUS, ENABLE-3S and their successors, VVM and SET Level ${ }^{1}$, address the issue of system validation and verification, following a scenariodriven approach.

Although simulation-based testing is broadly used throughout the development process [2], [3], real-world drivings unveil valuable details for further analysis. Therefore, however, relevant scenarios need to be extracted from, e.g., Naturalistic Driving Studies (NDS) or Field Operations Tests (FOT), which allows compiling an extensive knowledge base of scenarios [4] for comprehensive simulation-based testing.

This work addresses the issue of building up a knowledge base of scenarios focussing on the urban domain and proposes a novel methodology for routing scenario extraction. That is, the focus is on how motorized road users cross

\footnotetext{
* Lars Klitzke, Clemens Schicktanz and Kay Gimm are with the German Aerospace Center (DLR), Institute of Transportation Systems, Braunschweig, Germany, \{lars.klitzke, clemens.schicktanz, kay.gimm\}@dlr.de.

Carsten Koch is with the Hochschule Emden/Leer, University of Applied Sciences, Emden, Germany, carsten.kochehs-emden-leer.de.

FFrank Köster is with the German Aerospace Center (DLR), Institute for AI Safety and Security, Sankt Augustin / Ulm, Germany, frank. koesterddlr.de.

${ }^{1}$ www.pegasusprojekt.de/en, www.enable-s3.eu, www.vvm-projekt.de/en, https://setlevel.de/en
}

intersections. Therefore, the road user's conducted routing maneuver is inferred solely based on the association of the road user's trajectory to a route of a semantically enriched network. For that purpose, the work proposes to annotate roads of intersections in a road network with routing maneuvers as depicted in Fig. 1. The left image shows the German Aerospace Center (DLR) Application Platform for Intelligent Mobility (AIM) Research Intersection [5] with the OpenDRIVE reference lines color-encoded w.r.t their annotated routing maneuver, and the right image the intersection's topology with the annotated roads depicted as green circles.

A data-driven approach using clustering methods is proposed to annotate roads with primitive routing maneuvers w.r.t their shape. Furthermore, a route backtracking approach is proposed based on map-matching to estimate a road user's traveled route on intersections by extending a previously [6] proposed method. The semantically enriched road network and the backtracked route enable inferring a road user's maneuver robustly even in complex mixed traffic environments with various routes and thus maneuver candidates.

The efficacy of the road annotation approach is evaluated with the road network of Brunswick's the inner-city ring [7], which is available in OpenDRIVE [8] format. In addition, to demonstrate the consistency of the methodology, u-turn scenarios are extracted on the DLR AIM Research Intersection in Brunswick depicted in Fig. 1.

The paper is organized as follows. An overview of related work is given in Section II. The proposed methodology for road annotation with routing maneuvers is presented in Section III. This a priori knowledge about the maneuver is employed in Section IV to backtrack the route of road users on intersections for scenario extraction. An evaluation follows in Section V, showing the efficacy of the simple but yet effective approach and the consistency of the methodology. In Section VI the paper concludes with a discussion and outlook on further works.

\section{RELATED WORK}

Estimating the route of road users has been a broadly discussed problem for several decades. However, the approaches typically differ in that they look at traffic and the road network from either a microscopic or macroscopic point of view. For the latter, the road network's level-of-detail is typically lower than the former one or not relevant for the use-case at hand so that intersections are typically left out, and the network only contains interconnecting roads [9], [6].

A solution for the microscopic route estimation problem is mimicking the inductive loops that are embedded in 

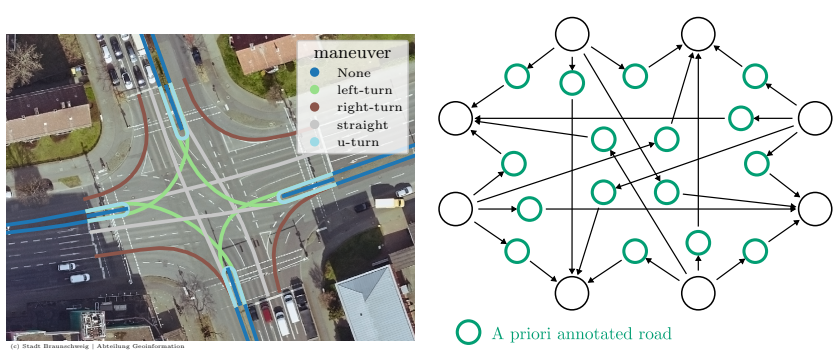

Fig. 1: The DLR Research Intersection in Brunswick. Left: The OpenDRIVE reference lines are depicted, colorized w.r.t the annotated maneuver. Right: The topology of the intersection as graph with the incoming and outgoing roads.

streets for, e.g., vehicle counting [10]. Therefore, virtual inductive loops are created manually according to the work's objectives. For example, Chen et al. create virtual loops as polygons to determine routes [11]. Junghans et al. use them on intersections to analyze unprotected left turns in mixed-traffic environments [12], and Dotzauer et al. in an online bicycle warning system [13]. Liu, Zeng and Jiang employ virtual loops for a real-time vehicle-counting system [14]. Although this method allows a flexible creation and adaptation to the work's objectives, scaling it for multiple regions is impractical due to the manual loop definition.

Another established technique for route estimation is map matching. The trajectories of road users are projected onto a digital representation of the road network. This mapped trajectory allows inferring the traveled route [9]. For that purpose, various approaches were proposed for online and offline map-matching using, for instance, Hidden Markov Model (HMM) [15], [9], [16] or geometric properties [17] of the road network. However, the works typically have a macroscopic view of traffic and the road network by neglecting intersection in the road network. But, a road-level view is required for microscopic traffic behavior analysis on intersections. Hansson et al. use an HMM for lane-level map matching based on GPS measurements, visual features of the surrounding and change indication information by on-board systems of the vehicle [18]. However, they show that the performance decreases with the absence of the lane-changing indicator signal, which is not available in this work, and that the transition probability matrix needs to be tailored to the digital map. Due to that, and since this work's overall aim is to extract driving scenarios in the urban domain, a mapmatching-based route estimation approach for microscopic traffic analysis on the road instead of lane level is proposed utilizing a digital map in OpenDRIVE format. For route estimation, a road is represented with the reference line instead of the lane borders as in [18], since each road on an intersection typically represents a driving direction in OpenDRIVE. Thus, the route can be estimated by mapping trajectories on reference lines. Moreover, the topology of the road network is employed for route estimation and thus maneuver annotation as depicted in Fig. 1 since it allows finding the possible routes on an intersection.

\section{ROAD NETWORK ANNOTATION}

As already described in the beginning, this work proposes to infer the maneuver that a traffic participant performs on an intersection, based on an a priori annotated digital map. For that purpose, this work employs the divide and conquer strategy [19]. That is, the road network, the complex space, will be divided into more trivial subspaces: the primitive maneuvers. By assuming that traffic participants use intersections to change their direction towards their destination, or not in case of crossing an intersection straight, this directional change that the traffic participant experience is used for clustering. Since only the digital representation of the road network will be used, it is assumed that the directional change is also represented in the shape of the OpenDRIVE reference lines. Therefore, a top-down clustering approach is proposed assuming that roads in a certain category have a common shape. In the following, the overall method for road network maneuver annotation is presented.

\section{A. Problem definition}

At first, let us assume that traffic participants either cross an intersection by driving straight through it or they change their driving direction towards their destination. Hence, the set of roads of an intersection can be partitioned into two sets, straight roads and turning roads. Furthermore, the set of turning roads is further divided into three subsets denoting the maneuvers: left, right and u-turn as depicted in Fig. 3 . Note that, even if a physical road is partially used to perform multiple routing maneuvers, the work assumes there exists virtual roads, so that each (virtual) road can be exclusively associated with a routing maneuver.

Formally defined, let $W_{N}=\left\{w_{1}, w_{2}, \ldots, w_{n}\right\}$ be the roads of an urban road network $N$. That network has subsets representing specific primitive routing maneuvers and is thus denoted as a semantic network $\hat{N}$. At first, the network $N$ has two disjoint proper subsets $N=\{A, B\}$ with $A=\left\{A_{1}, A_{2}, \ldots\right\}$ denoting the intersections and $B=\left\{B_{1}, B_{2}, \ldots\right\}$ denoting the roads connecting intersections. An intersection $I \in A$ is thus a set of $m$ roads $I=\left\{w_{1}, w_{2}, \ldots, w_{m}\right\}$ with $m<n$. The two subsets $C, D$ of $I$ are two disjoint sets, i.e., $C \cap D=\emptyset$. The set $C$ consists of the roads driving straight and $D$ of turning roads. To represent the three different variants of turning maneuver, left, right and u-turn, the set $D$ is divided into the subsets $E \subseteq F, F \subseteq D, G \subseteq D$. Note that all three sets are disjoint $(D \cap E \cap F)=\emptyset$ but are no proper subsets of $D$. The latter allows that an intersection might not contain all road types. Hence, an intersection $I \in \hat{N}$ of a semantic network $\hat{N}$ can be partitioned into the subsets $\{C, E, F, G\}$ as depicted in Fig. 2 as a dendrogram with $C$ denoting straight lines, $E$ left turns, $F$ right turns and $G$ u-turns.

\section{B. Feature estimation}

To annotate roads of an intersection with the primitive routing maneuver, and in particular of intersections, the aim is to find a function that associates the road $w$ with the correct subset of the semantic network $\hat{N}$, i.e., to find its semantic meaning in context of routing maneuvers. For that purpose, 


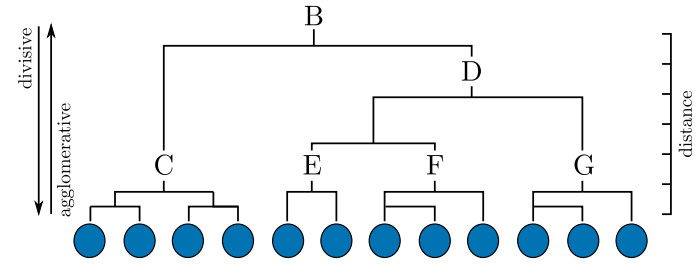

Fig. 2: An intersection partitioned into subsets shown as a dendrogram. Each subset represents a road type according to the routing maneuver that a road user performs.

this work employs a data-driven approach using unsupervised learning methods. That is, it is assumed that roads in a category are similar and can be robustly differentiated to roads of other categories. Therefore, appropriate features need to be derived from the road's geometry.

Since the primitive routing maneuvers addressed in this work define the directional change of a traffic participant crossing the intersection, e.g., left or right turn, this information is used for clustering. If we assume that $\kappa \in[-\pi, \pi]$ denotes an approximation of the signed curvature of a road, it is easy to verify that turning and non-turning lanes differ significantly in the magnitude of $\kappa$. This also holds for left turn and u-turn roads with the latter having a greater deviation than the former one. Apparently, the sign of $\kappa$ allows differentiating between left turn and right turn roads.

To estimate the curvature $\kappa$ for each road, the previously assumed characteristic that a directional change is applied to a traffic participant crossing the intersection w.r.t the maneuver is used. That is, the road's heading at the start and end point are compared to derive the orientation change and thus the curvature of the road. Therefore, for each road $w \in\left\{I_{1}^{N}, I_{2}^{N}, \ldots\right\}$ on every intersection $I_{N}$ of the road network $N$, the unit vectors $\mathbf{u}, \mathbf{v}$ at the start and end are estimated, i.e., the incoming and outgoing vectors for each road. Since this work employs a line segment based approximation of the road's geometry, the unit vectors at the start and end of each road equals to the unit vectors of the first and last line segment. The signed orientation difference $\theta\left(\mathbf{u}_{w}, \mathbf{v}_{w}\right)$ between $\mathbf{u}_{w}=\left[u_{1}, u_{2}\right]^{T}$ and $\mathbf{v}_{w}=\left[v_{1}, v_{2}\right]^{T}$ is used to define the road's curvature $\kappa(w)=\theta\left(\mathbf{u}_{w}, \mathbf{v}_{w}\right)$ defined as

$$
\theta\left(\mathbf{u}_{w}, \mathbf{v}_{w}\right)=\arctan 2\left(\operatorname{det}\left(\left[\mathbf{u}_{w} \mathbf{v}_{w}\right]\right), \mathbf{u}_{w}^{T} \mathbf{v}_{w}\right), \theta \in[-\pi, \pi]
$$

to ensure a four-quadrant and signed solution since the road's unit vectors can be arbitrarily placed in $\mathbb{R}^{2}$.

For the DLR research intersection, the start and end unit vectors for each road, as well as the road's curvature are depicted in Fig. 3. Roads associated with a driving straight maneuver $W_{C}$ have a curvature close to zero $\kappa(w) \approx 0, w \in$ $W_{C}$. Left $W_{E}$ and right turning roads $W_{F}$ have a higher curvature magnitude $|\kappa(a)| \gg|\kappa(b)|, a \in\left\{W_{E}, W_{F}\right\}, b \in W_{C}$ and differ in the sign, whereas u-turn roads have the highest curvature magnitude. Hence, the chosen feature satisfies the requirements that roads in the same category are similar but can be differentiated from roads of other categories.

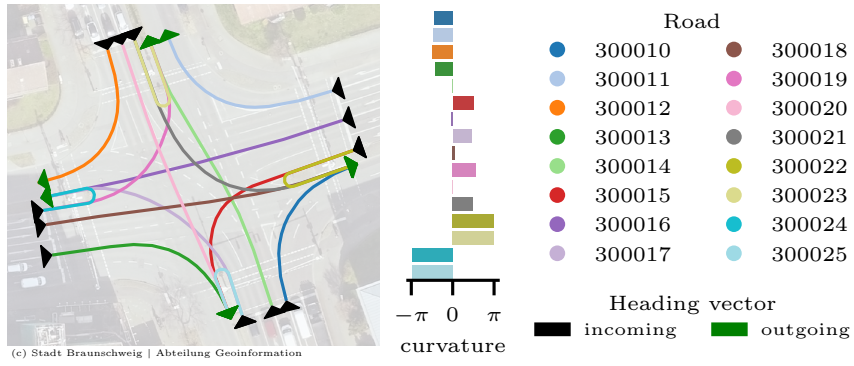

Fig. 3: The example intersection of the Brunswick inner-city road network. Left: A satellite image with the reference lines for each road. The incoming and outgoing vectors are shown for each road. Right: The road curvature distribution.

\section{Road network clustering}

Based on the derived feature, the aim is to associate each road to one of the maneuvers w.r.t its curvature. Since the number of clusters is known, partitioning or hierarchical clustering methods can be employed. However, the road network has to contain at least one road per maneuver category for both types. That might not be the case for a single intersection. However, if we assume that the network is sufficiently large, we can further assume at least one road per maneuver category is present.

For road maneuver annotation, this work proposes to cluster the network first and recover the correct maneuver type afterward. The latter is required since clustering methods might not return the same result on each execution time. Therefore, let $\hat{N}$ denote the semantic network after clustering. Then, each road's absolute curvature is employed to, at first, partition the road network into three clusters $\hat{N}=\{C, H, G\}$ for the maneuvers straight, left/right turn, and u-turn. The absolute curvature distribution for the intersection roads of the Brunswick inner-city road network is depicted in Fig. 4. The distribution contains three clusters at $\kappa \approx\left\{0, \frac{\pi}{2}, \pi\right\}$ representing the three maneuver clusters.

Let us assume that a clustering method divides the set of roads in the network $N$ into three clusters $\mathcal{X}=\left\{X_{1}, X_{2}, X_{3}\right\}$. The index $i=\{1,2, \ldots,|\hat{N}|\}$ of the subset $X_{i} \in N$ shall denote the correct maneuver. That index is estimated according to the expected curvature $E(X)=\frac{1}{|X|} \sum_{i=1}^{|X|}\left|\kappa_{i}\right|$ so that $U=$ $\left\{E\left(X_{1}\right), E\left(X_{2}\right), \ldots, E\left(X_{n}\right)\right\}$ with $n=|\mathcal{X}|$ denotes the expected value for each subset $X \in \mathcal{X}$. The correct maneuver class is inferred by sorting $U$ in ascending order so that $L=$ $\operatorname{argsort} U$ returns the indices $L=i_{1}, \ldots, i_{n}$ of the correct subset in $\hat{N}$. Finally, to differentiate between left $E$ and right turn roads $F$ in the set $H=\{E, F\}$, the sign of the curvature is employed such that $E=\left\{w \mid w \in H \wedge \kappa_{w}>0\right\}$ and $F=\left\{w \mid w \in H \wedge \kappa_{w}<0\right\}$. Since the overall approach is dependent on the used clustering algorithm, an evaluation of established methods is performed in Section V using the road network of Brunswick.

\section{ROUTE BACKTRACKING}

Given the semantic road network and the road user's trajectory, the traveled route must be estimated for routing 
scenario extraction. Therefore, map matching is employed to find the roads of a digital road network the traffic participant travels on [20]. The work proposes an approach for route backtracking depicted in Fig. 5, which will be presented in the following.

\section{A. Problem definition}

Let $X=\mathbf{x}_{1}, \mathbf{x}_{2}, \mathbf{x}_{3}, \ldots$ be the trajectory as a sorted series of poses of the traffic participant. A pose $\mathbf{x}=[u, v, \omega]^{T}$ is a vector representing the state of the object at a specific point in time. The object's location is denoted as $(u, v)$ in Universal Transverse Mercator (UTM) reference system. The object's orientation is given as $\omega \in(-\pi, \pi]$ with $\omega=0$ pointing east, increasing in counter-clockwise direction.

Furthermore, let $I=\{W, R, \psi\}$ be an intersection of the road network since the aim is to estimate the most likely route on intersections for maneuver annotation. $W=$ $\left\{w_{1}, w_{2}, \ldots\right\}$ is a set of roads of the intersection. Each road $w=\{S, \imath, m\}$ is represented as a series of line segments $S$ defining the geometry of the road, a unique identifier $l$, and an optional maneuver $m$ annotated with the approach described above. $R=\left\{r_{1}, r_{2}, \ldots\right\}$ is a set with all possible routes on that intersection derived from the topology of the road network. That is, a route $r \subset W$ represents a path of a road user to enter and leave the intersection with at most one road annotated with a routing maneuver. The connectivity between roads on the intersection is defined as set of tuples $\psi=\left\{\left(w_{i}, w_{j}\right) \mid w_{i} \in W, w_{j} \in W, w_{i} \neq w_{j}\right\}$ stating that road $w_{i}$ is connected to $w_{j}$ and no loops exist. In particular, $w_{j}$ is defined as the successor of $w_{i}$. Note that $\forall\left(w_{i}, w_{j}\right) \in \psi$ : $\left(w_{j}, w_{i}\right) \notin \psi$, i.e., roads have only one direction.

Since the aim is to annotate a traffic participant with a routing maneuver according to the road network, we need to find the route $r \in R_{I}$ of the intersection $I$ that the object follows. For that purpose, let us assume that $\mathcal{L}(r \mid \mathbf{x})$ is the likelihood of the pose $\mathbf{x} \in X$ mapped on the route $r \in R$. Then, the route backtracking problem can be written as a maximization problem

$$
\hat{r}=\underset{r}{\arg \max } \sum_{i=1}^{|X|} \mathcal{L}\left(r \mid \mathbf{x}_{i}\right)
$$

with $|X|$ denoting the number of poses in the trajectory and $\hat{r}$ as the most likely route.
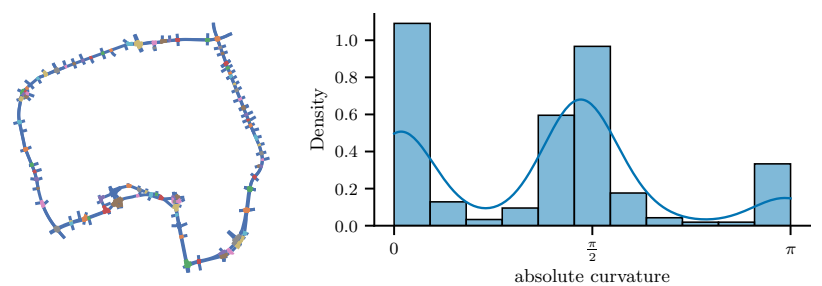

Fig. 4: The inner-city ring of Brunswick. Left: The network depicted with the OpenDRIVE reference lines. Intersections are color-encoded whereas interconnection roads are blue. Right: The curvature distribution for all intersection roads.
To solve the stated problem in (2), i.e., finding the most likely route a traffic participant follows on an intersection, this work employs a Directed Acyclic Graph of Mapped Roads (DAGMaR) presented in a previous work [6]. That is, by iteratively building up a Directed Acyclic Graph (DAG) with roads $R_{I}$ of the intersection $I$ according to the current state of the traffic participant $x \in X$, the likelihood being mapped on the roads $\mathcal{L}\left(w_{1} \mid \mathbf{x}\right), \mathcal{L}\left(w_{2} \mid \mathbf{x}\right), \ldots$ and the connectivity $\psi$ between roads of the intersection, the most likely route $\hat{r}$ is given by the longest path in the DAG. A major benefit of iteratively expanding the graph is that this allows to estimate the most likely route for each $x_{i} \in X$ given the preceding poses $x_{1}, \ldots, x_{i-1}$. Hence, the potentially conducted maneuver of a road user can be predicted.

\section{B. Likelihood models}

To estimate the route of a traffic participant using DAG$\mathrm{MaR}$, each pose $\mathbf{x} \in X$ of the trajectory will be mapped onto all roads in the vicinity of the current pose $\mathbf{x}$ as depicted in Fig. 5. This microscopic view on the road user's surrounding ensures that the graph will not over-expand and only relevant roads are considered for mapping.

Let $P_{\mathbf{x}}=\left\{\mathbf{p}_{1}, \mathbf{p}_{2}, \ldots, \mathbf{p}_{n}\right\}$ are the mapped poses of $\mathbf{x}$ to all $n$ roads in the vicinity $\mathcal{V}=\left\{w_{1}, w_{2}, \ldots, w_{n}\right\}$ of $\mathbf{x}$. A mapped pose $\mathbf{p}$ is defined as $\mathbf{p}=[u, v, \omega, \imath, \mathcal{L}]^{T}$ as an extension of $\mathbf{x}$ with $l$ as the identifier of the mapped $\operatorname{road} w \in \mathcal{V}$ and $\mathcal{L}$ as the likelihood of the pose being mapped on the road.

This work uses the deviation between the measured object pose $\mathbf{x}$ and all of its mapped poses $P_{\mathbf{x}}$ to estimate the road mapping likelihood. In particular, the euclidean distance $d(\mathbf{x}, \mathbf{p})$ and the orientation deviation $\Delta \omega(\mathbf{x}, \mathbf{p}) \in(-\pi, \pi]$ between the pose and its mapped siblings is used as in a previous work [6]. Hence $\mathcal{L}(w \mid \mathbf{p})$ is defined as

$$
\mathcal{L}(w \mid \mathbf{x})=\frac{\mathcal{L}(w \mid d(\mathbf{x}, \mathbf{p})) \mathcal{L}(w \mid \Delta \omega(\mathbf{x}, \mathbf{p}))}{\sum_{\tilde{w}}^{\mathcal{L}} \mathcal{L}(\tilde{w} \mid d(\mathbf{x}, \mathbf{p})) \mathcal{L}(\tilde{w} \mid \Delta \omega(\mathbf{x}, \mathbf{p}))}
$$

and thus the joint likelihood of $\mathcal{L}(w \mid d(\mathbf{x}, \mathbf{p}))$ and $\mathcal{L}(w \mid \Delta \omega(\mathbf{x}, \mathbf{p}))$, denoting the likelihood of $\mathbf{x}$ being mapped on $w \in \mathcal{V}$ given the euclidean distance and orientation deviation. The denominator in (3) ensures that $\sum_{w}^{\mathcal{V}} \mathcal{L}(w \mid \mathbf{x})=1$.

For the distance likelihood model $\mathcal{L}(w \mid d(\mathbf{x}, \mathbf{p}))$, the following requirements are defined. Since only the OpenDRIVE reference lines are used as geometric representation of each road, and they represent the leftmost border of the drivable area, each road's width needs to be integrated into the model. Furthermore, the model should allow to integrate uncertainty of the road width estimation and support the fact that traffic participants might not follow the digital representation of the road exactly but leave it in, for instance, curves. Due to that, the Tukey window [21] was adopted as depicted in Fig. 6 defined as a piecewise function

$$
\mathcal{L}(w \mid d)= \begin{cases}\frac{\left[1+\cos \left(\pi \frac{d+a_{1}}{b_{1}}\right)\right]}{2} & -\left(b_{1}+a_{1}\right) \leq d<-a_{1} \\ 1 & -a_{1} \leq d<a_{2} \\ \frac{\left[1+\cos \left(\pi \frac{d-a_{2}}{b_{2}}\right)\right]}{2} & a_{2} \leq d<\left(a_{2}+b_{2}\right) \\ \varepsilon & \text { else }\end{cases}
$$




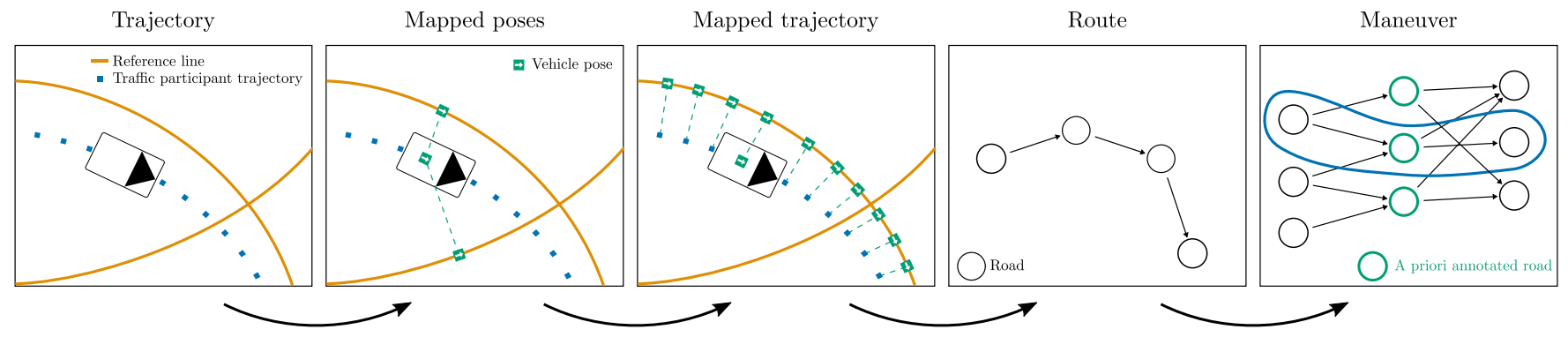

Fig. 5: Framework for map-driven routing maneuver annotation of traffic participants. The mapped trajectory is estimated using DAGMaR handling ambiguous situations robustly. The mapped trajectory is aggregated to a route of roads which is compared with all available paths on the intersection extracted from the maneuver-annotated logical road network. The routing maneuver is given by the most likely matched intersection route.

with $\mathcal{L}(w \mid d)$ as a short form of $\mathcal{L}(w \mid d(\mathbf{x}, \mathbf{p}))$ for the sake of readability. The parameters $a_{1}, a_{2}$ denote the deviation from the reference lane for the left and right side. The parameters $b_{1}, b_{2}$ allow to model deviation from the road that occur for instance in curves. Since it is assumed that a traffic participant usually drives at the right side of the reference line, $a_{2} \gg a_{1}$ and $b_{2} \gg b_{1}$ with $a_{2}$ is defined as the road's width and $b_{2}$ as a multiple of $a_{2}\left(a_{2}==b_{2}\right.$ in Fig. 6).

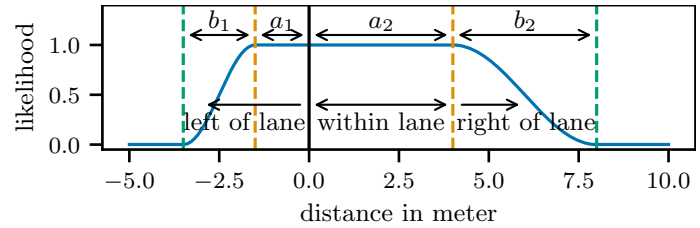

Fig. 6: The adopted Tukey window as the likelihood model for the distance to the road. The model supports to define the road width and decreasing likelihood for higher distances w.r.t the side of the road.

As for the distance likelihood model, requirements are defined for the orientation deviation model. At first, the likelihood needs to be inversely proportional to the orientation deviation. That is, the likelihood of being mapped on a road is higher if the orientation deviation is small and decreases with increasing deviation. Furthermore, the relationship between the orientation deviation increase and likelihood decrease should be non-linear to stronger penalize higher orientation deviations. Finally, the minimum likelihood given as a model parameter should be at $|\pi|$ since $\Delta \omega \in(-\pi, \pi]$, so that

$$
\min \mathcal{L}(w \mid \Delta \omega)=\mathcal{L}(w|\Delta \omega=| \pi \mid)=\varepsilon
$$

with $\varepsilon \in(0,1], \varepsilon \ll 1$ as the parameter defining the minimum likelihood at $|\Delta \omega|>\pi$. In this work, an exponentially decaying function is employed to model the orientation deviation likelihood, satisfying the defined criteria with

$$
\mathcal{L}(w \mid \Delta \omega)=\max \left\{\varepsilon, \frac{1}{e^{|\Delta \omega| \frac{\ln \frac{1}{\varepsilon}}{\pi}}}\right\}=\max \left\{\varepsilon, \varepsilon^{-\frac{|\Delta \omega|}{\pi}}\right\}
$$

as depicted in Fig. 7 with different values of $\varepsilon$ in linear (top) and logarithmic scale (bottom). It is clearly visible that changing $\varepsilon$ impacts the penalization of orientation deviations with a high penalization for smaller values of $\varepsilon$.

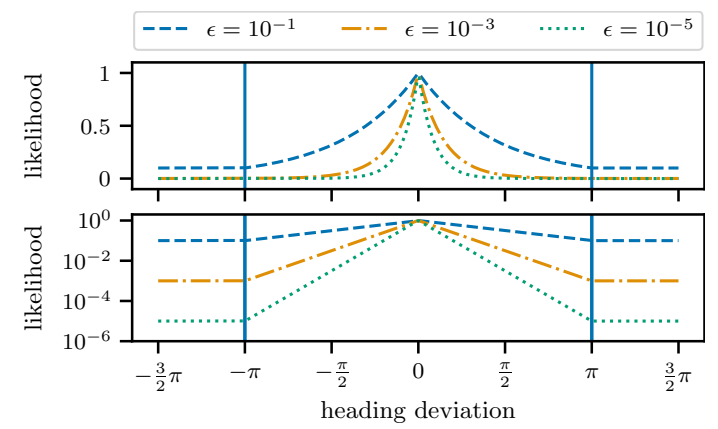

Fig. 7: An exponential decaying function is employed for the orientation deviation model. Top: The likelihood for different parameters. Bottom: The likelihood in log-space depicting that $\min \mathcal{L}(\Delta \omega \mid w)=\varepsilon$.

\section{Route estimation}

For finding the traveled route of the road user, the mapped poses are utilized to build up a DAGMaR since the most likely route $\hat{r} \in R$ is the longest path in DAGMaR. Therefore, the mapped poses are used with the likelihood models defined in (3) and (4) combined in (3). For further information about this approach, interested readers are encouraged to read [6]. For the sake of clarity, the approach is revisited briefly.

Let DAGMaR $D$ be defined analogously to the intersection $I$ as $D=\{W, \psi, M, I\}$ with $W=w_{1}, w_{2}, \ldots$ as the mapped roads and $M$ being an additional mapping $M=$ $\left\{\left(\mathbf{x}_{1}, P_{1}\right),\left(x_{2}, P_{2}\right), \ldots,\left(\mathbf{x}_{n}, P_{n}\right)\right\}$ of a pose $\mathbf{x}_{i}$ with its siblings $P_{i}=\left\{\mathbf{p}_{1}, \mathbf{p}_{2}, \ldots, \mathbf{p}_{m}\right\}$ mapped on the roads in the pose's vicinity $\mathcal{V}\left(\mathbf{x}_{i}\right)=\left\{w_{1}, w_{2}, \ldots, w_{m}\right\}$. Furthermore, since the aim is to backtrack the route on intersections, it's representation $I$ is part of DAGMaR. Moreover, each road $w \in W_{D}$ and connection $\left(w_{i}, w_{j}\right) \in \psi_{D}$ contains a weighting attribute that is used to find the longest path. Hence, DAGMaR is a weighted DAG.

If the road user enters an ambiguous state on an intersection I, i.e., there are multiple likely road mappings, DAGMaR is initialized to recover the route. For all following time-steps $t_{1}, t_{2}, \ldots$, the mapped poses $P_{t}$ of the traffic participant's pose 
$\mathbf{x}_{t}$ are added to DAGMaR as long as the traffic participant is within the intersection. For each added mapped pose, the road's likelihood is incremented. If the road user leaves the intersection, the actual route is backtracked, and based on that, the trajectory of mapped poses $\widetilde{X}=\left\{\tilde{\mathbf{p}}_{1}, \tilde{\mathbf{p}}_{2}, \ldots\right\}$ is recovered. Therefore, the longest path $\hat{r}=\left\{w_{1}, w_{2}, \ldots\right\}$ in DAGMaR is estimated. The final backtracked trajectory $\widetilde{X}$ is derived based on the route $\hat{r}$ and the mapping $M$ of DAGMaR $D$. Hence, the routing maneuver performed by the road user is given by the maneuver-annotated road on the route.

The next step is to identify the object's transition points between roads of a route. That allows analyzing the trajectory on specific ways. For that purpose, the backtracked trajectory $\widetilde{X}$ is divided into $n=|\hat{r}|$ subsets $\widetilde{X}_{1}, \widetilde{X}_{2}, \ldots, \widetilde{X}_{n}$ according to the number of ways in the most likely road $\hat{r}$. This is utilized in Section V-B, where road users performing u-turns on the research DLR research intersection are extracted, focussing on the trajectory part in the inner-intersection area.

\section{Evaluation}

The following section will discuss the presented approach for map-driven maneuver annotation and scenario extraction. Therefore, the road network annotation method is evaluated with the OpenDRIVE road network of Brunswick. Additionally, u-turn scenarios are extracted for proof-of-concept from a dataset collected on the DLR AIM research intersection.

\section{A. Road network maneuver annotation}

The dataset of Brunswick's inner-city ring contains 97 intersections with a total of 735 roads. The roads are manually annotated with a maneuver by inspecting the satellite image with the OpenDRIVE reference lines as shown in Fig. 1. The network contains 150 left-turn, 254 right-turn, 256 straight and 75 u-turn lanes.

The presented approach for road maneuver annotation employs clustering for grouping the roads according to their curvature. Thus, the results of different established clustering algorithms are evaluated. $k$-means, GMM and HAC are employed using the implementation in scikit-learn [22]. Since the performance of a GMM is dependent on the covariance type, different variants are analyzed. But, since the feature space is only one-dimensional, the performance should not differ significantly. For HAC, the linkage types average, complete, and ward are evaluated since they merge clusters according to different objectives [23][24].

The precision is estimated for each method and maneuver, i.e., the fraction of correctly labeled roads. Furthermore, the mean precision is calculated per algorithm. GMM with covariance type spherical performs best with a mean precision of $0.995 \pm 0.005$ and an overall accuracy of $99,72 \%$ since three roads were misclassified (see Table I). The results of HAC using linkage-type average and ward are similar and superior to the linkage-type complete. The latter lacks precision in left and right turn detection.

The three misclassified roads of GMM using the spherical coverage type are exemplarily depicted in Fig. 8. A rightturning road $F$ was incorrectly labeled as a straight lane $C$ for the left intersection. The straight lane $C$ in the middle image was annotated as a right turn lane $F$ and the left turn lane $E$ as a u-turn $G$ road in the right intersection.

$$
(F, C)
$$

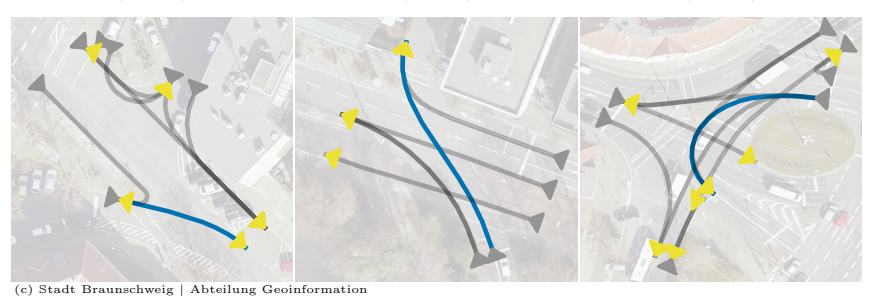

Fig. 8: The three misclassified roads by GMM (spherical). The title $(\cdot, \cdot)$ denotes the ground truth and estimated maneuver type as the cluster symbol in the semantic road network. The misclassified roads are highlighted in blue.

\section{B. U-turn scenario extraction}

The dataset used for evaluating the proposed scenario extraction approach was collected from $19^{\text {th }}$ until $26^{\text {th }}$ July 2021. The dataset contains 220.695 motorized road users. The proposed approach for scenario extraction $\mathrm{RB}$ is compared with using virtual inductive loops VL. Therefore, loops were manually created at the entries and exits of the intersection and only trajectories entering and leaving the intersection through the loops are considered. Trajectories are mapped to a route according to the combination of loop crossings. Only trajectories are considered valid for the

TABLE I: The clustering precision for $k$-means, Gaussian Mixture Model (GMM) with different covariance types and Hierarchical Agglomorative Clustering (HAC) with different linkage types. The precision is given as the ratio of correctly annotated roads to the total number of roads for each maneuver type.

\begin{tabular}{|c|c|c|c|c|c|c|}
\hline \multirow[b]{2}{*}{ Method } & \multirow[b]{2}{*}{ Variant } & \multicolumn{4}{|c|}{ Maneuver } & \multirow[b]{2}{*}{ Mean precision } \\
\hline & & Straight & Left turn & Right turn & U-turn & \\
\hline \multirow{5}{*}{$\begin{array}{l}k \text {-means } \\
\text { GMM }\end{array}$} & & 1.000 & 0.973 & 0.980 & 1.000 & $0.988 \pm 0.012$ \\
\hline & diag & 1.000 & 0.980 & 0.984 & 1.000 & $0.991 \pm 0.009$ \\
\hline & full & 1.000 & 0.980 & 0.984 & 1.000 & $0.991 \pm 0.009$ \\
\hline & spherical & 0.996 & 1.000 & 0.996 & 0.987 & $0.995 \pm 0.005$ \\
\hline & tied & 1.000 & 0.987 & 0.988 & 0.987 & $0.990 \pm 0.006$ \\
\hline \multirow[t]{3}{*}{$\mathrm{HAC}$} & average & 1.000 & 0.973 & 0.984 & 1.000 & $0.989 \pm 0.011$ \\
\hline & complete & 1.000 & 0.713 & 0.816 & 1.000 & $0.882 \pm 0.123$ \\
\hline & ward & 1.000 & 0.973 & 0.984 & 1.000 & $0.989 \pm 0.011$ \\
\hline
\end{tabular}


proposed approach $\mathrm{RB}$, with at least one mapped pose on each road of the associated route and fully traversing the semantically annotated road.

The results are depicted in Fig. 9, showing the number of extracted u-turn scenarios for the different regions. $R B$ can detect almost all scenarios. Both approaches identified 775 similar scenarios. VL extracted 21 different scenarios than $\mathrm{RB}$ and $\mathrm{RB} 22$ scenarios. The difference is due to the trajectory filtering approach. $\mathrm{RB}$ missed scenarios that did not follow the route, and VL failed to extract scenarios where trajectories start behind the virtual loops. That is, the approach's performance is sensitive to the location of the virtual loops. Fig. 9 shows the extracted u-turn scenarios of the proposed approach. Although the approach missed 21 u-turn scenarios, no false positives exist. Hence, the proposed methodology allows the extraction of u-turn scenarios robustly.

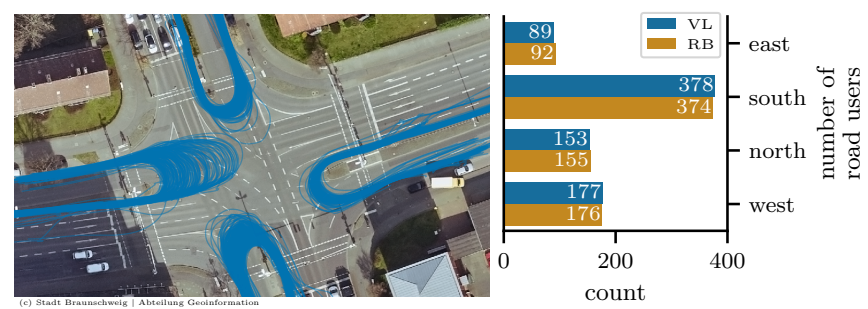

Fig. 9: The u-turn scenario extraction results. Left: The trajectories of the identified u-turn scenarios. Right: The number of road users per u-turn region extracted via virtual inductive loops $\mathrm{VL}$ and the proposed method $\mathrm{RB}$.

\section{CONCLUSION}

The method proposed in this work allows inferring primitive routing maneuvers based on the traffic participant's traveled route by enriching the roads of the digital representation of the road network with routing maneuvers. That allows to robustly extract scenarios which is valuable for the functional verification of AVs. The methodology contains two components. The first one annotates roads of intersections with primitive routing maneuvers. The second part uses this a priori knowledge to estimate a route user's road from a microscopic point of view. Combining both parts allows inferring road users routing maneuver based on the estimated route instead of its trajectory. The experiments have shown that the methodology robustly extracts u-turn scenarios on the DLR AIM Research Intersection in Brunswick.

Follow-up work will extend the proposed method to model routing maneuvers with interactions between road users, such as unprotected left-turns, including vulnerable road users. In addition, future work will perform a detailed analysis of $\mathrm{u}$ turn scenarios to explore the reasons for the high variability of the extracted u-turns, and to characterize u-turns on urban intersection for, e.g., simulation-based testing.

\section{REFERENCES}

[1] D. J. Fagnant and K. Kockelman, "Preparing a nation for autonomous vehicles: opportunities, barriers and policy recommendations," Transportation Research Part A: Policy and Practice, vol. 77, pp. 167-181, 2015.
[2] S. Hallerbach, Y. Xia, U. Eberle, and F. Köster, "Simulation-based identification of critical scenarios for cooperative and automated vehicles," SAE Intl. J CAV, vol. 1, pp. 93-106, 042018.

[3] C. Neurohr, L. Westhofen, M. Butz, M. H. Bollmann, U. Eberle, and R. Galbas, "Criticality analysis for the verification and validation of automated vehicles," IEEE Access, vol. 9, pp. 18 016-18 041, 2021.

[4] W. Damm, E. Möhlmann, T. Peikenkamp, and A. Rakow, "A formal semantics for traffic sequence charts," in Principles of Modeling. Springer, 2018, pp. 182-205.

[5] S. Knake-Langhorst and K. Gimm, "Aim research intersection: Instrument for traffic detection and behavior assessment for a complex urban intersection," Journal of large-scale research facilities JLSRF, vol. 2 , p. 65,2016

[6] L. Klitzke, J. Meyer, T. Leune, C. Koch, and F. Köster, "DAGMaR: A DAG-based Robust Road Membership Estimation Framework for Scenario Mining," in The Second International Workshop on Intelligent Transportation and Connected Vehicles Technologies (ITCVT 2019), Granada, Spain, Oct 2019, pp. 358-365.

[7] M. Scholz, "OpenDRIVE dataset of the inner ring road in Brunswick," Sept. 2020.

[8] ASAM. ASAM OpenDRIVE. Accessed: February 1, 2020. [Online]. Available: https://www.asam.net/standards/detail/opendrive/

[9] S. Taguchi, S. Koide, and T. Yoshimura, "Online map matching with route prediction," IEEE Transactions on Intelligent Transportation Systems, vol. 20, no. 1, pp. 338-347, 2019.

[10] P. J. Bickel, C. Chen, J. Kwon, J. Rice, E. van Zwet, and P. Varaiya, "Measuring traffic," Statistical Science, vol. 22, no. 4, 2007.

[11] P. Chen, W. Zeng, G. Yu, and Y. Wang, "Surrogate safety analysis of pedestrian-vehicle conflict at intersections using unmanned aerial vehicle videos," Journal of Advanced Transportation, vol. 2017, pp. $1-12,2017$.

[12] M. Junghans, F. Krauns, A. Sonka, M. Böhm, and M. Dotzauer, "Comparison of safety and kinematic patterns of automated vehicles turning left in interaction with oncoming manually driven vehicles," Transactions on Transport Sciences, 2021.

[13] M. Dotzauer, H. Saul, M. Junghans, K. Gimm, S. Knake-Langhorst, and C. Schieß1, "Online situation and risk assessment: Improving cyclists' safety in intersections?" 2018.

[14] F. Liu, Z. Zeng, and R. Jiang, "A video-based real-time adaptive vehicle-counting system for urban roads," PLOS ONE, vol. 12, no. 11, pp. 1-16, 112017.

[15] P. Newson and J. Krumm, "Hidden markov map matching through noise and sparseness," in Proceedings of the 17th ACM SIGSPATIAL international conference on advances in geographic information systems, 2009, pp. 336-343.

[16] C. Goh, J. Dauwels, N. Mitrovic, M. T. Asif, A. Oran, and P. Jaillet, "Online map-matching based on hidden markov model for realtime traffic sensing applications," in 2012 15th International IEEE Conference on Intelligent Transportation Systems, 2012, pp. 776-781.

[17] J. Rabe, M. Meinke, M. Necker, and C. Stiller, "Lane-level mapmatching based on optimization," in 2016 IEEE 19th International Conference on Intelligent Transportation Systems (ITSC), 2016, pp. $1155-1160$.

[18] A. Hansson, E. Korsberg, R. Maghsood, E. Nordn, and Selpi, "Lanelevel map matching based on HMM," IEEE Transactions on Intelligent Vehicles, vol. 6, no. 3, pp. 430-439, 2021.

[19] D. R. Smith, "The design of divide and conquer algorithms," Science of Computer Programming, vol. 5, pp. 37-58, 1985.

[20] Y. Hu and B. Lu, "A hidden markov model-based map matching algorithm for low sampling rate trajectory data," IEEE Access, vol. 7, pp. $178235-178245,2019$.

[21] J. W. Tukey, "An intoroduction to the calculation of numerical spectrum analysis," Spectra Analysis of Time Series, pp. 25-46, 1967.

[22] F. Pedregosa, G. Varoquaux, A. Gramfort, V. Michel, B. Thirion, O. Grisel, M. Blondel, P. Prettenhofer, R. Weiss, V. Dubourg, J. Vanderplas, A. Passos, D. Cournapeau, M. Brucher, M. Perrot, and E. Duchesnay, "Scikit-learn: Machine learning in Python," Journal of Machine Learning Research, vol. 12, pp. 2825-2830, 2011.

[23] N. Randriamihamison, N. Vialaneix, and P. Neuvial, "Applicability and interpretability of wards hierarchical agglomerative clustering with or without contiguity constraints," Journal of Classification, vol. 38, no. 2, pp. 363-389, 2021.

[24] O. Yim and K. T. Ramdeen, "Hierarchical cluster analysis: comparison of three linkage measures and application to psychological data," The quantitative methods for psychology, vol. 11, no. 1, pp. 8-21, 2015. 